\title{
EXISTENCE AND ASYMPTOTICS OF FRONTS IN NON LOCAL COMBUSTION MODELS*
}

\author{
ANTOINE MELLET ${ }^{\dagger}$, JEAN-MICHEL ROQUEJOFFRE ${ }^{\ddagger}$, AND YANNICK SIRE ${ }^{\S}$
}

Abstract. We prove the existence and provide the asymptotics for nonlocal fronts in homogeneous media.

Key words. Reaction-diffusion equations, fractional laplacian, asymptotic behaviour.

AMS subject classifications. 35A01, 35B08.

\section{Introduction}

This paper is devoted to the study of front propagation in homogeneous media for a fractional reaction-diffusion equation appearing in combustion theory. More precisely, we consider the following scalar model for the combustion of premixed gas with ignition temperature:

$$
u_{t}+\left(-\partial_{x x}\right)^{\alpha} u=f(u) \quad \text { in } \mathbb{R} \times \mathbb{R}
$$

where the function $f$ satisfies:

$$
\left\{\begin{array}{l}
f: \mathbb{R} \rightarrow \mathbb{R} \text { continuous function, } \\
f(u) \geq 0 \text { for all } u \in \mathbb{R} \text { and } \operatorname{supp} f=[\theta, 1] \\
f^{\prime}(1)<0
\end{array}\right.
$$

where $\theta \in(0,1)$ is a fixed number (usually referred to as the ignition temperature).

The operator $\left(-\partial_{x x}\right)^{\alpha}$ denotes the fractional power of the Laplace operator in one dimension (with $\alpha \in(0,1])$. It can be defined by the following singular integral

$$
\left(-\partial_{x x}\right)^{\alpha} u(x)=c_{\alpha} \mathrm{PV} \int_{\mathbb{R}} \frac{u(x)-u(z)}{|x-z|^{1+2 \alpha}} d z,
$$

where PV stands for the Cauchy principal value. This integral is well defined, for instance, if $u$ belongs to $C^{2}(\mathbb{R})$ and satisfies

$$
\int_{\mathbb{R}} \frac{|u(x)|}{(1+|x|)^{1+2 \alpha}} d x<+\infty
$$

(in particular, smooth bounded functions are admissible). Alternatively, the fractional Laplace operator can be defined as a pseudo-differential operator with symbol $|\xi|^{2 \alpha}$. We refer the reader to the book by Landkof where an extensive study of $\left(-\partial_{x x}\right)^{\alpha}$ is performed by means of harmonic analysis techniques (see [10]).

\footnotetext{
* Received: December 15, 2012; accepted (in revised form): December 15, 2012. Communicated by Lenya Ryzhik.

${ }^{\dagger}$ Department of Mathematics, Mathematics Building, University of Maryland, College Park, MD 20742-4015, USA (antoine.mellet@gmail.com).

${ }^{\ddagger}$ Institut de Mathmatiques, Universit Paul Sabatier, 118 route de Narbonne, 31062 Toulouse Cedex, France (jean-michel.roquejoffre@math.univ-toulouse.fr).

§"Universit Aix-Marseille, Centre de Mathmatique et Informatique, Laboratoire d'Analyse, Topologie, Probabilit (LATP), Technopole de Chateau-Gombert, 9, rue F. Joliot Curie, 13453 Marseille Cedex 13 France (sire@cmi.univ-mrs.fr).
} 
In this paper, we will always take $\alpha \in(1 / 2,1]$, and we are interested in particular solutions of (1.1) which describe transition fronts between the stationary states 0 and 1 (traveling fronts). These traveling fronts are solutions of (1.1) that are of the form

$$
u(t, x)=\phi(x+c t)
$$

with

$$
\left\{\begin{array}{l}
\lim _{x \rightarrow-\infty} \phi(x)=0 \\
\lim _{x \rightarrow+\infty} \phi(x)=1
\end{array}\right.
$$

The number $c$ is the speed of propagation of the front. It is readily seen that $\phi$ must solve

$$
\left(-\partial_{x x}\right)^{\alpha} \phi+c \phi^{\prime}=f(\phi), \quad \text { for all } x \in \mathbb{R}
$$

When $\alpha=1$ (standard Laplace operator), it is well known that there exists a unique speed $c$ and a unique profile $\phi$ (up to translation) that correspond to a traveling front solution of (1.1) (see e.g. $[9,3,4,5]$ ). The goal of this paper is to generalize these results to the case $\alpha \in(1 / 2,1)$. We are thus looking for $\phi$ and $c$ satisfying

$$
\left\{\begin{array}{l}
\left(-\partial_{x x}\right)^{\alpha} \phi+c \phi^{\prime}=f(\phi), \quad \text { for all } x \in \mathbb{R}, \\
\lim _{x \rightarrow-\infty} \phi(x)=0, \\
\lim _{x \rightarrow+\infty} \phi(x)=1, \\
\phi(0)=\theta
\end{array}\right.
$$

(the last condition is a normalization condition which ensures the uniqueness of $\phi$ ). Our main theorem is the following.

THEOREM 1.1. If $\alpha \in(1 / 2,1)$ and $f$ satisfies (1.2), then there exists a unique pair $\left(\phi_{0}, c_{0}\right)$ solving (1.5). Furthermore, $c_{0}>0$ and $\phi_{0}$ is monotone increasing.

We will also obtain the following result, which describes the asymptotic behavior of the front at $-\infty$.

THEOREM 1.2. Let $\alpha \in(1 / 2,1)$ and assume that $f$ satisfies (1.2). Let $\phi_{0}$ be the unique solution of (1.5) provided by Theorem 1.1. Then there exist $m, M$ such that

$$
\phi_{0}(x) \leq \frac{M}{|x|^{2 \alpha-1}} \quad \text { for } x \leq-1
$$

and

$$
\phi_{0}^{\prime}(x) \geq \frac{m}{|x|^{2 \alpha}} \quad \text { for } x \leq-1
$$

In view of these estimates, it is clear that the case $\alpha=1 / 2$ is critical since the decay of $\phi_{0}$ at infinity degenerates when $\alpha \rightarrow 1 / 2$. It is actually proved in [8] that the speed of propagation is unbounded when $\alpha<1 / 2$ (so no traveling wave can exist in that case). The case $\alpha=1 / 2$ is, to the authors knowledge, still open at this time (the method developed in this paper does not extend to that case). We also note that this dichotomy between the cases $\alpha<1 / 2$ and $\alpha>1 / 2$ when $f$ is the ignition temperature 
nonlinearity should be contrasted with the case of the Fisher-KPP type nonlinearities for which the speed of front propagation is unbounded for all $\alpha \in(0,1)$ (see $[6,8,7])$.

The proof of Theorem 1.1 follows classical arguments developed by BerestyckiLarrouturou-Lions [3] (see also Berestycki-Nirenberg [4]): Truncation of the domain, construction of sub- and super-solutions and passage to the limit. As usual, one of the main difficulty is to make sure that we recover a finite, non trivial speed of propagation at the limit. The main novelty (compared with similar results when $\alpha=1$ ) is the construction of sub- and super-solutions where the classical exponential profile is replaced by power tail functions.

As a remark, the stability of the waves is obtained in a standard way assuming some good decay at $-\infty$.

The paper is organized as follows: in a first section, we provide the truncation of the domain and study the associated problem. In a second section, we provide the proof of Theorem 1.1. Finally, a last section is devoted to the asymptotic properties of the wave.

\section{Truncation of the domain}

The first step is to truncate the domain: for some $b>0$, we consider the following problem:

$$
\left\{\begin{array}{l}
\left(-\partial_{x x}\right)^{\alpha} \phi_{b}+c_{b} \phi_{b}^{\prime}=f\left(\phi_{b}\right), \quad \text { for all } x \in[-b, b], \\
\phi_{b}(x)=0, \quad \text { for } s \leq-b, \\
\phi_{b}(x)=1, \quad \text { for } s \geq b, \\
\phi_{b}(0)=\theta .
\end{array}\right.
$$

The goal of this section is to prove that this problem has a solution for $b$ large enough. More precisely, we are now going to prove the following proposition.

Proposition 2.1. Assume $\alpha \in(1 / 2,1)$ and that $f$ satisfies (1.2). Then there exists a constant $M$ such that if $b>M$, then the truncated problem (2.1) has a unique solution $\left(\phi_{b}, c_{b}\right)$. Furthermore, the following properties hold:

(i) There exists $K$ independent of $b$ such that $-K \leq c_{b} \leq K$.

(ii) $\phi_{b}$ is nondecreasing with respect to $x$ and satisfies $0<\phi_{b}(x)<1$ for all $x \in$ $(-b, b)$.

Before we can prove this proposition, we need to detail the construction of suband super-solutions.

2.1. Construction of sub- and super-solutions. In the proof of the existence of traveling waves for the standard Laplace operator $(\alpha=1)$, sub- and supersolutions of the form $e^{\gamma x}$ play a crucial role, in particular in the determination of the asymptotic behavior of the traveling waves as $x \rightarrow-\infty$. These particular functions are replaced, in the case of the fractional Laplace operator, by functions with polynomial tail. In what follows, we will rely on the following two important lemmas.

Lemma 2.2. Let $\beta \in(0,1)$ and define

$$
\varphi(x)= \begin{cases}\frac{1}{|x|^{\beta}} & \text { if } x<-1 \\ 1 & \text { if } x>-1\end{cases}
$$

Then $\varphi$ satisfies

$$
\left(-\partial_{x x}\right)^{\alpha} \varphi+c \varphi^{\prime}(x)=\frac{-c_{\alpha}}{2 \alpha|x|^{2 \alpha}}+c \frac{\beta}{|x|^{\beta+1}}+O\left(\frac{1}{|x|^{\beta+2 \alpha}}\right)
$$


when $x \rightarrow-\infty$.

LEMMA 2.3. Let $\beta>1$ and define

$$
\bar{\varphi}(x)= \begin{cases}\frac{1}{|x|^{\beta}} & \text { if } x<-1 \\ 0 & \text { if } x>-1\end{cases}
$$

Then

$$
\left(-\partial_{x x}\right)^{\alpha} \bar{\varphi}+c \bar{\varphi}^{\prime}(x)=\frac{-c_{\alpha}}{\beta-1} \frac{1}{|x|^{2 \alpha+1}}+c \frac{\beta}{|x|^{\beta+1}}+O\left(\frac{1}{|x|^{\beta+2 \alpha}}\right)
$$

when $x \rightarrow-\infty$.

Proof. [Proof of Lemma 2.2.] We want to estimate $\left(-\partial_{x x}\right)^{\alpha} \varphi$ for $x<-1$. We have

$$
\left(-\partial_{x x}\right)^{\alpha} \varphi(x)=-c_{\alpha} \mathrm{PV} \int_{\mathbb{R}} \frac{\varphi(x+y)-\varphi(x)}{|y|^{1+2 \alpha}} d y
$$

which we decompose as follows:

$$
\begin{aligned}
\left(-\partial_{x x}\right)^{\alpha} \varphi(x) & =c_{\alpha} \int_{-\infty}^{-1-x} \frac{\varphi(x)-\varphi(x+y)}{|y|^{1+2 \alpha}} d y+c_{\alpha} \int_{-1-x}^{+\infty} \frac{\varphi(x)-\varphi(x+y)}{|y|^{1+2 \alpha}} d y \\
& =I+I I
\end{aligned}
$$

A simple explicit computation yields

$$
I I=\left(\frac{1}{|x|^{\beta}}-1\right) \frac{c_{\alpha}}{2 \alpha|x+1|^{2 \alpha}} .
$$

Performing the change of variables $y=x z$, one gets

$$
I=\frac{c_{\alpha}}{|x|^{\beta+2 \alpha}} \int_{+\infty}^{-\frac{1}{x}-1} \frac{|z+1|^{\beta}-1}{|z+1|^{\beta}|z|^{1+2 \alpha}} d z
$$

Note that the integrand has a singularity at $z=0$, and this integral has to be understood as a principal value. We also observe that the integrand has a singularity at $z=-1$, but since $\beta<1$, this singularity is integrable, and thus

$$
I \sim-c_{\alpha} \frac{1}{|x|^{\beta+2 \alpha}} \mathrm{PV} \int_{-1}^{+\infty} \frac{|z+1|^{\beta}-1}{|z+1|^{\beta}|z|^{1+2 \alpha}} d z . \quad \text { as } x \rightarrow-\infty .
$$

We deduce that

$$
\left(-\partial_{x x}\right)^{\alpha} \varphi(x)=\frac{-c_{\alpha}}{2 \alpha|x|^{2 \alpha}}+O\left(\frac{1}{|x|^{\beta+2 \alpha}}\right)
$$

when $x \rightarrow-\infty$, and the result follows.

Proof. [Proof of Lemma 2.3.] Again, we decompose $\left(-\partial_{x x}\right)^{\alpha} \bar{\varphi}$ as follows:

$$
\left(-\partial_{x x}\right)^{\alpha} \bar{\varphi}(x)=c_{\alpha} \int_{-\infty}^{-1-x} \frac{\bar{\varphi}(x)-\bar{\varphi}(x+y)}{|y|^{1+2 \alpha}} d y+c_{\alpha} \int_{-1-x}^{+\infty} \frac{\bar{\varphi}(x)-\bar{\varphi}(x+y)}{|y|^{1+2 \alpha}} d y
$$




$$
=I+I I
$$

Now, a simple explicit computation yields

$$
I I=\frac{c_{\alpha}}{|x|^{\beta}} \frac{1}{2 \alpha|x+1|^{2 \alpha}}
$$

Performing the change of variables $y=x z$, one gets

$$
I=\frac{c_{\alpha}}{|x|^{\beta+2 \alpha}} \int_{+\infty}^{-\frac{1}{x}-1} \frac{|z+1|^{\beta}-1}{|z+1|^{\beta}|z|^{1+2 \alpha}} d z .
$$

Note that the integrand as a singularity at $z=0$, and this integral must be understood as a principal value. We also observe that the integrand has a singularity at $z=-1$ and since $\beta>1$, this singularity is divergent and thus

$$
I \sim \frac{-c_{\alpha}}{\beta-1}|x|^{\beta-1}
$$

We deduce that

$$
\left(-\partial_{x x}\right)^{\alpha} \bar{\varphi}(x)=\frac{-c_{\alpha}}{\beta-1} \frac{1}{|x|^{2 \alpha+1}}+O\left(\frac{1}{|x|^{\beta+2 \alpha}}\right),
$$

which yields the result.

2.2. Proof of Proposition 2.1. We now turn to the proof of Proposition 2.1. First, we fix $c \in \mathbb{R}$ and consider the following problem:

$$
\begin{cases}\left(-\partial_{x x}\right)^{\alpha} \phi+c \phi^{\prime}=f(\phi), & \text { for all } x \in[-b, b] \\ \phi(x)=0, & \text { for } x \leq-b \\ \phi(x)=1, & \text { for } x \geq b\end{cases}
$$

We have the following lemma.

LEMma 2.4. For any $c \in \mathbb{R}$, Equation (2.2) has a unique solution $\phi_{c}$. Furthermore $\phi_{c}$ is nondecreasing with respect to $x$ and $c \mapsto \phi_{c}$ is continuous.

Proof. Since 1 and 0 are respectively super- and sub-solutions, we can use Perron's method (recall that the fractional laplacian enjoys a comparison principle) to prove the existence of a solution $\phi_{c}(x)$ for any $c \in \mathbb{R}$. By a sliding argument, we can show that $\phi_{c}$ is unique and nondecreasing with respect to $x$. The fact that the function $c \mapsto \phi_{c}$ is continuous follows from classical arguments (see [4] for details).

We now have to show that there exists a unique $c=c_{b}$ such that $\phi_{c_{b}}(0)=\theta$. This will be a consequence of the following lemma.

LEMMA 2.5. There exist constants $M, K$ such that for $b>M$ the following properties hold:

1. If $c>K$ then the solution of (2.2) satisfies $\phi_{c}(0)<\theta$,

2. If $c<-K$ then the solution of (2.2) satisfies $\phi_{c}(0)>\theta$.

Together with the fact that $\phi_{c}(0)$ is continuous with respect to $c$, Lemma 2.5 implies that there exists $c_{b} \in[-K,-K]$ such that $\phi_{c_{b}}$ satisfies $\phi_{c_{b}}(0)=\theta$ and is thus a solution of (2.1). This completes the proof of Proposition 2.1. 
Proof. [Proof of Lemma 2.5.] We consider the function

$$
\varphi(x)= \begin{cases}\frac{1}{|x|^{2 \alpha-1}} & \text { if } x<-1 \\ 1, & \text { if } x \geq-1\end{cases}
$$

and note that Lemma 2.2 (with $\beta=2 \alpha-1$ ) yields that if $c$ is large enough ( $c \geq$ $\left.\frac{c_{\alpha}}{2 \alpha(2 \alpha-1)}\right)$, then

$$
\left(-\partial_{x x}\right)^{\alpha} \varphi(x)+c \varphi^{\prime}(x) \geq 0
$$

for $x \leq-A$ (for some $A$ large enough). We can also assume that $\varphi(x) \leq \theta$ for $x \leq-A$, and so

$$
\left(-\partial_{x x}\right)^{\alpha} \varphi(x)+c \varphi^{\prime}(x) \geq f(\varphi)=0 \quad \text { for } x \leq-A .
$$

Furthermore, for $-A<x<-1,\left(-\partial_{x x}\right)^{\alpha} \varphi(x)$ is bounded while

$$
c \varphi^{\prime}(x) \geq c \frac{2 \alpha-1}{A^{2 \alpha}} .
$$

For $c$ large enough, we thus have

$$
\left(-\partial_{x x}\right)^{\alpha} \varphi(x)+c \varphi^{\prime}(x) \geq \sup f \geq f(\varphi) \quad \text { for }-A<x<-1 .
$$

We deduce that there exists $K$ such that if $c \geq K$ then

$$
\left(-\partial_{x x}\right)^{\alpha} \varphi(x)+c \varphi^{\prime}(x) \geq f(\varphi) \quad \text { for } x<-1,
$$

and so $\varphi$ is a supersolution for (2.2).

Choosing $M$ such that $\varphi(-M)<\theta$, we now see that if $c \geq K$ and $b>M$, then $\varphi(x-M)$ is a super-solution for (2.2). By a sliding argument, we deduce that $\phi_{c}(x) \leq$ $\varphi(x-M)$ and so $\phi_{c}(0) \leq \varphi(-M)<\theta$.

For the lower bound, we define $\varphi_{1}(x)=1-\varphi(-x)$. Then we have, if $-c \geq K$ $(c \leq-K)$ and for $x>1$,

$$
\left(-\partial_{x x}\right)^{\alpha} \varphi_{1}(x)+c \varphi_{1}^{\prime}(x)=-\left[\left(-\partial_{x x}\right)^{\alpha} \varphi(-x)+(-c) \varphi^{\prime}(-x)\right] \leq 0 \leq f(\varphi) .
$$

Moreover, we have $\varphi_{1}(x)=0$ for $x \leq 1$. Proceeding as above, we deduce that if $c \leq-K$, then $\phi_{c}(0)>\theta$, which concludes the proof.

\section{Proof of Theorem 1.1}

In order to complete the proof of Theorem 1.1, we have to prove that we can pass to the limit $b \rightarrow \infty$ in the truncated problem. More precisely, Theorem 1.1 follows from the following proposition.

Proposition 3.1. Under the conditions of Proposition 2.1, there exists a subsequence $b_{n} \rightarrow \infty$ such that $\phi_{b_{n}} \longrightarrow \phi_{0}$ and $c_{b_{n}} \longrightarrow c_{0}$. Furthermore, $c_{0} \in(0, K]$ and $\phi_{0}$ is a monotone increasing solution of (1.5).

Proof of Proposition 3.1. We recall that $c_{b} \in[-K, K]$, and classical elliptic estimates (see [1]) yield

$$
\left\|\phi_{b}\right\|_{\mathcal{C}^{2, \gamma}} \leq C
$$


for some $\gamma \in(0,1)$. Thus there exists a subsequence $b_{n} \rightarrow \infty$ such that

$$
\begin{gathered}
c_{n}:=c_{b_{n}} \longrightarrow c_{0} \in[-K, K], \\
\phi_{n}:=\phi_{b_{n}} \longrightarrow \phi_{0},
\end{gathered}
$$

as $n \rightarrow \infty$. It is readily seen that $\phi_{0}$ solves

$$
\left(-\partial_{x x}\right)^{\alpha} \phi_{0}+c_{0} \phi_{0}^{\prime}=f\left(\phi_{0}\right), \quad \text { for all } x \in \mathbb{R} .
$$

It is also readily seen that $\phi_{0}(x)$ is monotone increasing, $\phi_{0}(0)=\theta$ and $\phi_{0}$ is bounded. By a standard compactness argument, there exists $\gamma_{0}, \gamma_{1}$ such that $\lim _{x \rightarrow-\infty} \phi_{0}(x)=\gamma_{0}$ and $\lim _{x \rightarrow+\infty} \phi_{0}(x)=\gamma_{1}$, with

$$
0 \leq \gamma_{0} \leq \theta \leq \gamma_{1} \leq 1
$$

It remains to prove that $c_{0}>0, \gamma_{0}=0$ and $\gamma_{1}=1$. For that, we will mainly follow classical arguments (see $[3,2]$ ).

First, we have the following lemma.

LEMma 3.2. The function $\phi_{0}$ satisfies

$$
\int_{\mathbb{R}}\left(-\partial_{x x}\right)^{\alpha} \phi_{0}(x) d x=0 .
$$

Proof. The result follows formally by integrating formula (1.3) with respect to $x$ and using the antisymmetry with respect to the variables $x$ and $z$. However, because of the principal value, one has to be a little bit careful with the use of Fubini's theorem.

To avoid this difficulty, we will use instead the equivalent formula for the fractional laplacian:

$$
\begin{aligned}
\left(-\partial_{x x}\right)^{\alpha} \phi_{0}(x)= & c_{\alpha} \int_{\mathbb{R} \backslash[x-\varepsilon, x+\varepsilon]} \frac{\phi_{0}(x)-\phi_{0}(z)}{|x-z|^{1+2 \alpha}} d z \\
& +c_{\alpha} \int_{[x-\varepsilon, x+\varepsilon]} \frac{\phi_{0}(x)-\phi_{0}(z)+\phi_{0}^{\prime}(x)(z-x)}{|x-z|^{1+2 \alpha}} d z
\end{aligned}
$$

which is valid for all $\varepsilon>0$ and does not involve singular integrals. Integrating the first term with respect to $x \in \mathbb{R}$, and using Fubini's theorem, we get

$$
\begin{aligned}
\int_{\mathbb{R}} \int_{\mathbb{R} \backslash[x-\varepsilon, x+\varepsilon]} \frac{\phi_{0}(x)-\phi_{0}(z)}{|x-z|^{1+2 \alpha}} d z d x & =\int_{\mathbb{R}} \int_{\mathbb{R} \backslash[z-\varepsilon, z+\varepsilon]} \frac{\phi_{0}(x)-\phi_{0}(z)}{|x-z|^{1+2 \alpha}} d x d z \\
& =-\int_{\mathbb{R}} \int_{\mathbb{R} \backslash[x-\varepsilon, x+\varepsilon]} \frac{\phi_{0}(x)-\phi_{0}(z)}{|x-z|^{1+2 \alpha}} d z d x,
\end{aligned}
$$

and so this integral vanishes. Using Taylor's theorem, the second term in (3.2) can be rewritten as

$$
\int_{x-\varepsilon}^{x+\varepsilon} \frac{1}{|x-z|^{1+2 \alpha}} \int_{x}^{z}(z-t) \phi_{0}^{\prime \prime}(t) d t d z=\int_{-\varepsilon}^{\varepsilon} \frac{1}{|y|^{1+2 \alpha}} \int_{x}^{x+y}(y+x-t) \phi_{0}^{\prime \prime}(t) d t d y .
$$

Integrating with respect to $x$ and using (twice) Fubini's theorem, we deduce

$$
\int_{\mathbb{R}} \int_{x-\varepsilon}^{x+\varepsilon} \frac{1}{|x-z|^{1+2 \alpha}} \int_{x}^{z}(z-t) \phi_{0}^{\prime \prime}(t) d t d z d x
$$




$$
\begin{aligned}
& =\int_{-\varepsilon}^{\varepsilon} \frac{1}{|y|^{1+2 \alpha}} \int_{-\infty}^{+\infty} \int_{x}^{x+y}(y+x-t) \phi_{0}^{\prime \prime}(t) d t d x d y \\
& =\int_{-\varepsilon}^{\varepsilon} \frac{1}{|y|^{1+2 \alpha}} \int_{-\infty}^{+\infty} \int_{t-y}^{t}(y+x-t) \phi_{0}^{\prime \prime}(t) d x d t d y \\
& =\int_{-\varepsilon}^{\varepsilon} \frac{y^{2}}{2|y|^{1+2 \alpha}} \int_{-\infty}^{+\infty} \phi_{0}^{\prime \prime}(t) d t d y \\
& =0
\end{aligned}
$$

where we used the fact that $\lim _{x \rightarrow \pm \infty} \phi_{0}^{\prime}(x)=0$ and so $\int_{-\infty}^{+\infty} \phi_{0}^{\prime \prime}(t) d t=0$. The lemma follows.

Now, we can integrate Equation (3.1) with respect to $x \in \mathbb{R}$, and using Lemma 3.2 , we get

$$
\int_{\mathbb{R}} f\left(\phi_{0}(x)\right) d x=c_{0}\left(\gamma_{1}-\gamma_{0}\right)<\infty .
$$

In particular, we observe that (3.3) implies that

$$
f\left(\gamma_{0}\right)=f\left(\gamma_{1}\right)=0,
$$

otherwise the integral would be infinite.

Next, we prove the following lemma.

Lemma 3.3. The limiting speed satisfies

$$
c_{0}>0
$$

Proof. First of all, we note that for all $n$, there exists $a_{n} \in\left(0, b_{n}\right)$ such that $\phi_{n}\left(a_{n}\right)=\frac{1+\theta}{2}$. Furthermore, up to another subsequence, by elliptic estimates, the function $\psi_{n}(x)=\phi_{b_{n}}\left(a_{n}+x\right)$ converges to a function $\psi_{0}$. Note that since $\psi_{0} \in \mathcal{C}^{\gamma}$, there exists $r>0$ such that

$$
\psi_{0}(x) \in\left[\frac{3+\theta}{4}, \frac{1+3 \theta}{4}\right] \quad \text { for } x \in[-r, r]
$$

and so there exists $\kappa_{0}>0$ such that

$$
\int_{\mathbb{R}} f\left(\psi_{0}\right) d x>\kappa_{0} .
$$

Up to a subsequence, we can assume that $b_{n}+a_{n}$ is either convergent or goes to $+\infty$. We need to distinguish between the two cases.

Case 1: $b_{n}+a_{n} \rightarrow+\infty$ : In this case, $\psi_{0}$ solves

$$
\left(-\partial_{x x}\right)^{\alpha} \psi_{0}+c_{0} \psi_{0}^{\prime}=f\left(\psi_{0}\right) \quad \text { for all } x \in \mathbb{R} .
$$

Furthermore, $\psi_{0}(0)=\frac{1+\theta}{2}$ and $\psi_{0}$ is monotone increasing. In particular, it is readily seen that there exists $\bar{\gamma}_{0}$ and $\bar{\gamma}_{1}$ such that $\lim _{x \rightarrow-\infty} \psi_{0}(x)=\bar{\gamma}_{0}$ and $\lim _{x \rightarrow+\infty} \psi_{0}(x)=$ $\bar{\gamma}_{1}$, with

$$
0 \leq \bar{\gamma}_{0} \leq \frac{1+\theta}{2} \leq \bar{\gamma}_{1} \leq 1
$$


Integrating (3.5) over $\mathbb{R}$, and using the fact that

$$
\int_{\mathbb{R}}\left(-\partial_{x x}\right)^{\alpha} \psi_{0}(x) d x=0
$$

(the proof is the same as in Lemma 3.2), we deduce

$$
c_{0}\left(\bar{\gamma}_{1}-\bar{\gamma}_{0}\right)=\int_{\mathbb{R}} f\left(\psi_{0}\right) d x<\infty,
$$

and so

$$
f\left(\bar{\gamma}_{0}\right)=f\left(\bar{\gamma}_{1}\right)=0 .
$$

This implies that

$$
\bar{\gamma}_{1}=1 \quad \text { and } \quad \bar{\gamma}_{0} \leq \theta .
$$

Finally, (3.6) and (3.4) yields

$$
c_{0}(1-\theta) \geq \int_{\mathbb{R}} f\left(\psi_{0}\right) d x \geq \kappa_{0},
$$

which gives the result.

Case 2: $a_{n}+b_{n} \rightarrow \bar{a}<\infty$ : In this case, $\psi_{0}$ solves

$$
\left(-\partial_{x x}\right)^{\alpha} \psi_{0}+c_{0} \psi_{0}^{\prime}=f\left(\psi_{0}\right) \quad \text { for all } x \in(-\infty, \bar{a}),
$$

and we need to modify the proof slightly. First, we notice that $\psi_{0}(x)=1$ for $x \geq \bar{a}$, and we observe that $\left(-\partial_{x x}\right)^{\alpha} \psi_{0}(x) \geq 0$ for $x \geq \bar{a}$. In particular,

$$
\int_{-\infty}^{\bar{a}}\left(-\partial_{x x}\right)^{\alpha} \psi_{0}(x) d x \leq \int_{\mathbb{R}}\left(-\partial_{x x}\right)^{\alpha} \psi_{0}(x) d x=0 .
$$

Proceeding as above, we check that $\lim _{x \rightarrow-\infty} \psi_{0}(x)=\bar{\gamma}_{0} \leq \theta$, and integrating (3.7) over $(-\infty, \bar{a})$, we deduce

$$
c_{0}(1-\theta) \geq \int_{\mathbb{R}} f\left(\psi_{0}\right) d x>0 .
$$

The positivity of the speed, together with the sub-solution constructed in Lemma 2.2 will now give $\gamma_{0}=0$. More precisely, we now prove the following lemma.

LEMMA 3.4. The function $\phi_{0}$ satisfies

$$
\lim _{x \rightarrow-\infty} \phi_{0}(x)=0 .
$$

Proof. Let $c_{1}=c_{0} / 2>0$ and take $n$ large enough so that $c_{b_{n}} \geq c_{1}$.

We recall that by Lemma 2.2 (see also the proof of Lemma 2.5), the function

$$
\varphi(x)= \begin{cases}\frac{1}{|x|^{2 \alpha-1}} & \text { if } x<-1 \\ 1 & \text { if } x>-1\end{cases}
$$


satisfies

$$
\left(-\partial_{x x}\right)^{\alpha} \varphi+K \varphi^{\prime} \geq 0 \quad \text { in }\{\varphi<1\},
$$

for some $K$ large enough. Introducing $\varphi_{\varepsilon}(x)=\varphi(\varepsilon x)$, we deduce

$$
\left(-\partial_{x x}\right)^{\alpha} \varphi_{\varepsilon}+\varepsilon^{2 \alpha-1} K \varphi_{\varepsilon}^{\prime}(x) \geq 0 \quad \text { in }\left\{\varphi_{\varepsilon}(x)<1\right\},
$$

and taking $\varepsilon$ small enough (recalling that $2 \alpha>1$ ), we get

$$
\left(-\partial_{x x}\right)^{\alpha} \varphi_{\varepsilon}+c_{1} \varphi_{\varepsilon}^{\prime}(x) \geq 0 \quad \text { in }\left\{\varphi_{\varepsilon}<1\right\} .
$$

Furthermore, $\varphi_{\varepsilon}=1$ for $x \geq 0$, and so by a sliding argument, we deduce $\phi_{b_{n}}(x) \leq \varphi_{\varepsilon}(x)$ for all $n$ such that $c_{b_{n}} \geq c_{1}$, and thus

$$
\phi_{0}(x) \leq \varphi_{\varepsilon}(x),
$$

which implies in particular that $\gamma_{0}=0$.

Finally, we conclude the proof of Proposition 3.1 by proving that $\gamma_{1}=1$.

LEMMA 3.5. The function $\phi_{0}$ satisfies

$$
\lim _{x \rightarrow+\infty} \phi_{0}(x)=1
$$

Proof. We recall that (3.3) implies that either $\gamma_{1}=\theta$ or $\gamma_{1}=1$ (otherwise the integral is infinite). Furthermore, if $\gamma_{1}=\theta$, then $\phi_{0} \leq \theta$ on $\mathbb{R}$ and so $\int_{\mathbb{R}} f\left(\phi_{0}(x)\right) d x=0$. Since $\gamma_{0}=0<\theta$, (3.3) implies $c_{0}=0$, which is a contradiction. Hence $\gamma_{1}=1$.

\section{Asymptotic behavior}

We now prove Theorem 1.2, which further characterizes the behavior of $\phi_{0}$ as $x \rightarrow-\infty$. We recall that in the case of the regular Laplace operator $(\alpha=1), \phi_{0}$ and its derivatives decrease exponentially fast to 0 as $x \rightarrow-\infty$. When $\alpha \in(1 / 2,1)$, it is readily seen that the proof of Lemma 3.4 actually implies the following.

Proposition 4.1 (Asymptotic behavior of $\phi_{0}$ ). There exists $M$ such that

$$
\phi_{0}(x) \leq \frac{M}{|x|^{2 \alpha-1}} \quad \text { for } x \leq-1 .
$$

Noticing that $\phi_{0}^{\prime}>0$ solves

$$
\left(-\partial_{x x}\right)^{\alpha} \phi_{0}^{\prime \prime}+c_{0}\left(\phi_{0}^{\prime}\right)^{\prime}=0 \quad \text { for } x \leq 0,
$$

we can also prove the following result.

Proposition 4.2 (Asymptotic behavior of $\phi_{0}^{\prime}$ ). There exists a constant $m$ such that

$$
\phi_{0}^{\prime}(x) \geq \frac{m}{|x|^{2 \alpha}} \quad \text { for } x \leq-1
$$


Proof. Lemma 2.3 implies that the function

$$
\bar{\varphi}(x)= \begin{cases}\frac{1}{|x|^{2 \alpha}} & \text { if } x<-1, \\ 0 & \text { if } x>-1\end{cases}
$$

satisfies

$$
\left(-\partial_{x x}\right)^{\alpha} \bar{\varphi}+c \bar{\varphi}^{\prime}(x)=-\frac{c_{\alpha}}{2 \alpha-1} \frac{1}{|x|^{2 \alpha+1}}+c \frac{2 \alpha}{|x|^{2 \alpha+1}}+O\left(\frac{1}{|x|^{4 \alpha}}\right)
$$

when $x \rightarrow \infty$, and so

$$
\left(-\partial_{x x}\right)^{\alpha} \bar{\varphi}+k \bar{\varphi}^{\prime}(x) \leq 0 \quad \text { for } x \leq-A
$$

if $k$ is small enough and $A$ is large.

We introduce $\varphi_{\varepsilon}(x)=\bar{\varphi}(\varepsilon x)$, which satisfies

$$
\left(-\partial_{x x}\right)^{\alpha} \varphi_{\varepsilon}+\varepsilon^{1-2 \alpha} k \varphi_{\varepsilon}^{\prime} \leq 0 \quad \text { for } x<-\varepsilon^{-1} A,
$$

hence

$$
\left(-\partial_{x x}\right)^{\alpha} \varphi_{\varepsilon}+c_{0} \varphi_{\varepsilon}^{\prime} \leq 0 \quad \text { for } x<-\varepsilon^{-1} A
$$

provided we choose $\varepsilon$ small enough.

Finally, we take $r$ so that

$$
\phi_{0}^{\prime}(x) \geq r \varphi_{\varepsilon}(x) \quad \text { for }-\varepsilon^{-1} A<x<-\varepsilon^{-1} .
$$

Proposition 4.2 now follows from the maximum principle and a sliding argument using the fact that $\varphi_{\varepsilon}(x)=0$ for $x \geq-\varepsilon^{-1}$.

\section{REFERENCES}

[1] J.-M. Bony, P. Courrège, and P. Priouret, Semi-groupes de Feller sur une variété à bord compacte et problèmes aux limites intégro-différentiels du second ordre donnant lieu au principe du maximum, Ann. Inst. Fourier (Grenoble), 18(fasc. 2), 369-521, 1969.

[2] H. Berestycki and F. Hamel, Generalized travelling waves for reaction-diffusion equations, Contemp. Math., Amer. Math. Soc., Providence, RI, 446, 101-123, 2007.

[3] H. Berestycki, B. Larrouturou, and P.L. Lions, Multi-dimensional travelling-wave solutions of a flame propagation model, Arch. Rat. Mech. Anal., 111(1), 33-49, 1990.

[4] H. Berestycki and L. Nirenberg, Travelling fronts in cylinders, Ann. Inst. H. Poincaré Anal. Non Linéaire, 9(5), 497-572, 1992.

[5] H. Berestycki, B. Nicolaenko, and B. Scheurer, Traveling wave solutions to combustion models and their singular limits, SIAM J. Math. Anal., 16(6), 1207-1242, 1985.

[6] X. Cabré and J.-M. Roquejoffre, Propagation de fronts dans les équations de Fisher-KPP avec diffusion fractionnaire, C.R. Math. Acad. Sci. Paris, 347(23-24), 1361-1366, 2009.

[7] A.-C. Coulon and J.-M. Roquejoffre, Transition between linear and exponential propagation in fisher-kpp type reaction-diffusion equations, Comm. PDEs, to appear.

[8] H. Engler, On the speed of spread for fractional reaction-diffusion equations, Int. J. Diff. Equ., $16,2010$.

[9] J.I. Kanel, Stabilization of solutions of the Cauchy problem for equations encountered in combustion theory, Mat. Sb. (N.S.), 59 (101), 245-288, 1962.

[10] N.S. Landkof, Foundations of Modern Potential Theory, Springer-Verlag, New York, 1972. 